\title{
Dental Health State Utility Values Associated with Compromised First Permanent Molar Teeth
}

\section{Dr Aisling Cant ( $\sim$ aisling.cant@nhs.net )}

Paediatric Dentistry, Centre of Oral, Clinical and Translational Science, Faculty of Dentistry, Oral \& Craniofacial Sciences, King's College London, London

Dr Risha Sanghvi

Paediatric Dentistry, Centre of Oral, Clinical and Translational Science, Faculty of Dentistry, Oral \& Craniofacial Sciences, King's College London, London

\section{Dr Aline de Almeida Neves}

Paediatric Dentistry, Centre of Oral, Clinical and Translational Science, Faculty of Dentistry, Oral \& Craniofacial Sciences, King's College London, London

\section{Professor Marie Therese Hosey}

Paediatric Dentistry, Centre of Oral, Clinical and Translational Science, Faculty of Dentistry, Oral \& Craniofacial Sciences, King's College London, London

\section{Professor Avijit Banerjee}

Centre of Oral, Clinical and Translational Sciences / Conservative \& MI Dentistry, Faculty of Dentistry, Oral \& Craniofacial Sciences, King's College London

\section{Fiona Warburton}

Oral Clinical Research Unit, Faculty of Dentistry, Oral \& Craniofacial Sciences, King's College London, London

\section{Dr Mark Pennington}

King's Health Economics, Institute of Psychiatry, Psychology \& Neuroscience, King's College London, London

\section{Research Article}

Keywords: Compromised first permanent molar teeth, Utility values

Posted Date: June 28th, 2021

DOI: https://doi.org/10.21203/rs.3.rs-646093/v1

License: (우 (i) This work is licensed under a Creative Commons Attribution 4.0 International License. Read Full License 


\section{Abstract}

\section{Background}

The aim of this study was to generate utility data to support economic evaluation of interventions for compromised first permanent molars (cFPMs) and to assess the reliability of responses using alternative elicitation methods.

\section{Methods}

Fifty adult respondents attending a Paediatric Dentistry department with their child completed a questionnaire to elicit utility values for eight clinical treatment scenarios for a compromised first permanent molar tooth (cFPM). These scenarios included restorations, prosthetic replacements or presence of a gap (missing tooth). The question formats included a ranking, a visual analogue scale (VAS) and a time trade off (TTO) exercise.

\section{Results}

Mean utility values for each health state were generated using the VAS and TTO data. A white filling generated the highest utility ( 0.786 [VAS], 0.763 [TTO]) and was ranked as the most favourable treatment option. An open gap generated the lowest utility ( 0.344 [VAS], 0.523 [TTO]). A closed gap (0.6392 [VAS]) was rated below various tooth restorations but above a traditional bridge (0.5944 [VAS]) or denture (0.4312 [VAS]), according to mean VAS scores. VAS responses showed better agreement (kappa $=0.48)$ with the ranking exercise compared to TTO responses $(k a p p a=-0.003)$.

\section{Conclusions}

Plausible and broadly consistent values were generated with VAS and TTO, but TTO data seemed to be less reliable. These data allow comparison of treatment options involving extraction and gap closure or restoration of cFPMs using a single measure of patient preference.

\section{Background}

Economic evaluation is now firmly established as a tool to support decision making in medicine. The value of economic evaluation has been enhanced through the development and application of a single measure, the Quality Adjusted Life-year (QALY), to quantify health benefits across almost the entire gamut of medical interventions and treatments (1). Under certain assumptions, QALYs are a measure of the utility or wellbeing derived from a change in health. In contrast to medicine, application of economic evaluation in dentistry has been limited. One of the rate limiting factors is the absence of measures to estimate the utility associated with dental health states (2).

The QALY combines longevity with health-related quality of life (QoL) rated on a scale where one represents full or maximum health and zero represents death (3). Typically, health-related QoL is assigned using a questionnaire, such as the EQ-5D-5L, that assesses the level of health functioning on a limited number of domains; under assumptions of independence across domains the level of functioning for each domain contributes to a global score or 'tariff' based on population survey values (4). In principle, these measures could be applied to dental interventions (5). However, this is rare in practice, reflecting a perception that they lack sensitivity to changes in oral health. Such a conclusion would be supported by the results of mapping from the OHIP-14, a measure of oral health quality of life, to EQ-5D-3L, which indicated a poor overlap between instruments with regard to the domains of health-related QoL (6). Domains such as aesthetics, which are central to preferences on dental interventions, are at best captured obliquely in general health-related QoL measures.

Quantification of health-related QoL requires measurement of preferences over health states. These preferences can be elicited directly using techniques such as visual analogue scale (VAS), time trade-off (TTO) and standard gamble (SG). The VAS approach requires respondents to mark a point on a line indicating the utility or value attached to a specific health state. The ends of the line are typically demarcated as best and worst conceivable health. Utilities are calculated as the fraction of the line from worst possible health, and then typically weighted to generate a value of zero for an anchor state (typically death). The TTO approach necessitates a choice between a period of time in a specific health state and a shorter period of time in full health followed by a period in the anchor state (again typically death). The utility is the ratio of the duration in full health considered equivalent to the to the duration in the 
respective health state. In the SG approach, respondents report the maximum risk of a worst outcome (typically death) they would entertain to gain a chance of the best health outcome compared to certainty of the respective health state. The risk is the disutility associated with the health state. VAS is perceived to be less demanding cognitively than TTO or SG but suffers from biases whereby respondents may avoid the ends of the VAS scale and seek to space out values to different health states. TTO, along with discrete choice experiments, has been the method of choice for recent valuation exercises for the EuroQoL EQ-5D-5L (7).

Researchers have attempted to measure preferences associated with different health states for an individual tooth. Such measures allow construction of a quality-adjusted tooth-year or QATY, which under certain assumptions allows quantification of the utility associated with different tooth health states (8). Valuation techniques applied to the elicitation of utilities for oral health states have included visual analogue scale (VAS), standard gamble (SG) and time trade off (TTO) (2). An early economic analysis of treatments for periodontal disease used TTO and SG exercises to determine weights for anterior and posterior teeth with different levels of sensitivity and acceptability of aesthetics (9). Results were reported in terms of cost per QATY for alternative treatments, assuming that the utility associated with different states of oral health is a simple sum of the utility assigned to each tooth in the respective mouth. Standard gamble has been used to generate utility scores for a posterior tooth in four health states ranging from decayed and painful to permanently restored (10). Utility values for dentine regeneration, root canal treatment (RCT) and extraction have been reported based on a SG exercise (11).

Dental caries and hypomineralisation defects are the most common reasons for the poor prognosis of first permanent molar teeth in children (12). Extraction at an appropriate age allows for closure of the gap left by the extraction and may provide acceptable functional and aesthetic outcomes $(13,14)$. Whilst preservation of an aesthetically acceptable, pain-free and functional tooth seems ideal, the longer-term outcomes of keeping the tooth include the risk of subsequent tooth loss and less acceptable restorations. The utility provided by tooth and prosthodontic restorations are essential to determine whether extraction or restoration of a compromised first permanent molar (cFPM) provides superior patient outcomes.

This study aimed to generate utility values for a first permanent molar tooth to inform an economic evaluation comparing extraction with restoration as treatment options in children with one or more cFPMs. The results of a survey in which TTO and VAS were used to elicit utility values for eight health states potentially arising from treatment choices for a cFPM are reported.

\section{Methods}

This study was conducted in the Paediatric Dentistry Department at St Thomas' Hospital, London and approved by NHS research ethics, namely HRA and Health and Care Research Wales (HCRW) (REC reference 19/NS/0164). Participants were provided with an invitation letter and an information leaflet regarding the study, and a written consent form was signed by the parent/carer if they agreed to participate. Consent was voluntary and participation was on an opt-in basis only. The participant could withdraw consent at any time.

The study aimed to recruit 50 parents/carers of children from those who were referred to the new patient assessment clinic. To avoid selection bias, all eligible parents/carers were approached on the clinic. The following exclusion criteria were applied: the child referred was under 3 or over 16 years of age; the child had medical problems, hypodontia (missing teeth) or had cFPMs which required extraction; the parent had insufficient knowledge of English. These exclusions were applied to avoid the influence that certain medical and dental conditions might have on participant's values. Parents of children with cFPMs were excluded, to avoid the influence this questionnaire may have had on their treatment preferences for their child's cFPMs.

The questionnaire comprised an introduction, a ranking exercise, questions eliciting preferences using VAS and TTO and questions on demographics. Guidance was followed on the development of questionnaires for eliciting preferences over health states (15). The questionnaire was piloted for content and face validity among colleagues and patients. The questionnaire and associated consent material were shown to several parents attending the Paediatric Dentistry clinic. Patient feedback was considered regarding their layout and content. The Gunning Fog Index was used to ensure appropriate readability. The reading age for the study documents ranged from age 7 - 10 years. Parents found the study documents easy to read and found the layout acceptable. Regarding the questionnaire, one parent found 'some of it a little complicated'. One parent stated that they had to 'read it a few times before they could understand it'. Following this feedback, we ensured that the researcher would be available to help participants if they had any questions. 
Eight health states were valued in the questionnaire representing the most common outcomes of either restoring or extracting a cFPM over the long term:

- White filling

- Silver crown

- Root canal treatment and crown

- Open gap (tooth missing)

- Closed gap (tooth missing)

- Extraction and bridge

- Extraction and implant

- Extraction and denture.

The reader is directed to the original questionnaire in Additional File 1 for a brief description of the eight health states.

\section{Ranking exercise}

Participants were asked to imagine if they had a cFPM. Different treatment options were presented in a table with an explanation of each treatment option. The participant was asked to rank these treatment options from 1 (most preferred option) to 8 (least preferred option).

\section{VAS method}

Participants were asked to think about the same treatment options. For each option, the participant was asked to rate it on a $100 \mathrm{~mm}$ scale from 0 to 100. The ends of the scale were labelled as the worst possible outcome and the best possible outcome.

\section{TTO method}

The participant was presented with a choice between a traditional treatment outcome and a hypothetical treatment that would result in a perfectly healthy tooth but with reduced longevity. The traditional treatment option would last for 10 years, after which any tooth or restoration would be removed, and a gap would remain. The hypothetical treatment would last for less than ten years after which the tooth would be removed and a gap would remain. Each participant was asked how many years (out of 10 years) with a 'perfect tooth' followed by a gap would be equivalent to the traditional treatment option.

The questionnaire concluded with questions on age, gender, ethnic background, employment status and partial postcode. The questionnaire took approximately 10 minutes to complete. The parent completed the questionnaire after the child's dental appointment. Questionnaires were administered with guidance and support from the researcher to help ensure respondents understood the tasks and to promote engagement with the survey.

\section{Statistical Analysis}

Response data were summarised as means, medians, standard deviations, and inter quartile range of scores. The response data was checked for normality using histograms. The data were skewed and therefore the relationship between demographic characteristics and responses were examined using either the Mann Whitney $U$ test or the Kruskal Wallis test, with significance set at $p<0.05$. Since this was an exploratory analysis no adjustment was made for multiple testing. Weighted kappa was used to assess the agreement between the ranking, VAS and TTO responses. All statistical analysis of the data was carried out using Stata version $16 \AA$ and Excel®.

\section{Results}

Two hundred and twenty-nine parents were approached, of which 127 were excluded. Of the remaining 102 parents, 47 declined to participate and five were not included following attainment of the target sample size of 50 participants (Figure 1).

Table 1 summarizes the participant characteristics. Most participants were aged between 35 and 44 years. Most participants were female and two thirds were currently employed $(n=32)$. A wide variation in ethnic background was noted, consistent with the local 
patient cohort.

Table 1: Demographic characteristics of respondents

\begin{tabular}{|ll|}
\hline Demographic characteristics & Number (\%) \\
\hline Age (years) & $8(16 \%)$ \\
\hline $25-34$ & $26(52 \%)$ \\
\hline $35-44$ & $15(30 \%)$ \\
\hline $45-54$ & $1(2 \%)$ \\
\hline Prefer not to say & \\
\hline & \\
\hline Gender & $30(60 \%)$ \\
\hline Female & $16(32 \%)$ \\
\hline Male & $4(8 \%)$ \\
\hline Prefer not to say & \\
\hline & \\
\hline Employment & $32(64 \%)$ \\
\hline Working & $13(26 \%)$ \\
\hline Not working & $5(10 \%)$ \\
\hline Prefer not to say & \\
\hline & \\
\hline Ethnic Background & $19(38 \%)$ \\
\hline White British & $(10 \%)$ \\
\hline Other White & \\
\hline Indian & \\
\hline Black African & \\
\hline Other & \\
\hline & \\
\hline
\end{tabular}

A white filling was ranked the best state by 26 participants. The open gap state and denture state were reported as the least favourable state by 22 and 19 participants, respectively (Figure 2). Figure 3 reports the mean ranking of each state. Notably, the closed gap, bridge and implant supported crown states had similar mean ranking values below that of a restored tooth and above the open gap and denture states.

Table 2 summarizes the VAS response data. As with the ranking data, the white filling state was rated highest and the open gap and denture states lowest. Differences in mean values for the remaining states were modest.

Table 2: Results of each health state using VAS method 


\begin{tabular}{|c|c|c|c|c|c|c|}
\hline & Mean & $\begin{array}{l}\text { Utility } \\
\text { Value }\end{array}$ & $\begin{array}{l}\text { Standard } \\
\text { Deviation }\end{array}$ & Median & $\begin{array}{l}\text { First quartile }\left(25^{\text {th }}\right. \\
\text { percentile) }\end{array}$ & $\begin{array}{l}\text { Third quartile }\left(75^{\text {th }}\right. \\
\text { percentile) }\end{array}$ \\
\hline White filling & 78.6 & 0.786 & 3.3386 .12 & 90 & 70.75 & 95 \\
\hline Silver Crown & 63.12 & 0.6312 & 3.8575 & 70 & 50 & 81.25 \\
\hline RCT and Crown & 65.84 & 0.6584 & 3.7085 & 69 & 50 & 90 \\
\hline Extraction and Gap & 34.38 & 0.3438 & 3.8719 & 30 & 10 & 51.25 \\
\hline $\begin{array}{l}\text { Extraction and Gap } \\
\text { Closes }\end{array}$ & 63.92 & 0.6392 & 3.9895 & 70 & 44.75 & 90 \\
\hline Extraction and Bridge & 59.44 & 0.5944 & 3.579 & 60 & 40 & 80 \\
\hline $\begin{array}{l}\text { Extraction and } \\
\text { Implant }\end{array}$ & 66.4 & 0.664 & 3.7307 & 70 & 48.5 & 90 \\
\hline $\begin{array}{l}\text { Extraction and } \\
\text { Denture }\end{array}$ & 43.12 & 0.4312 & 4.3305 & 39 & 18 & 71.25 \\
\hline
\end{tabular}

Table 3 summarizes the TTO response data. Again, the white filling state was rated highest and the denture and open gap states rated low. However, the TTO responses indicated a low valuation for the closed gap state. The valuation of the open gap state provides an indication of the validity of responses as any duration of time with a perfect tooth followed by an open gap ought to be preferable to the open gap state. Only 9 respondents provided TTO valuations of 1 year or less for this state suggesting most respondents had not understood the exercise.

Table 3 Results of each health state using TTO method

\begin{tabular}{|lllllll|}
\hline & Mean & $\begin{array}{l}\text { Utility } \\
\text { Value }\end{array}$ & $\begin{array}{l}\text { Standard } \\
\text { Deviation }\end{array}$ & Median & $\begin{array}{l}\text { First quartile (25 } \\
\text { percentile) }\end{array}$ & $\begin{array}{l}\text { Third quartile (75th } \\
\text { percentile) }\end{array}$ \\
\hline White Filling & 7.63 & 0.763 & 0.2927 & 8 & 6.75 & 9 \\
\hline Silver Crown & 6.96 & 0.696 & 0.3365 & 8 & 6 & 8.5 \\
\hline RCT and Crown & 6.88 & 0.688 & 0.3475 & 8 & 5 & 9 \\
\hline $\begin{array}{l}\text { Extraction and Gap } \\
\text { Extraction and Gap }\end{array}$ & 5.23 & 0.523 & 0.4217 & 5 & 2.75 & 8 \\
\hline $\begin{array}{l}\text { Closes } \\
\text { Extraction and Bridge }\end{array}$ & 6.82 & 0.555 & 0.4616 & 6 & 2 & 8 \\
\hline $\begin{array}{l}\text { Extraction and } \\
\text { Implant }\end{array}$ & 6.91 & 0.682 & 0.3428 & 7 & 6 & 8.125 \\
\hline $\begin{array}{l}\text { Extraction and } \\
\text { Denture }\end{array}$ & 5.99 & 0.599 & 0.3702 & 6.5 & 6.75 & 8 \\
\hline
\end{tabular}

The order of preferences for the VAS scores and TTO scores were compared with the ranking exercise scores for each participant. Weighted kappa scores indicated a closer match between the ranking scores and the VAS responses (Table 4).

Table 4: Agreement of preferences between ranking and VAS/TTO responses 


\begin{tabular}{|llllll|}
\hline Ranking vs. VAS & \multicolumn{5}{c|}{ Ranking vs. TTO } \\
\hline Method & Kappa (wgt) & Agreement & Method & Kappa (wgt) & Agreement \\
\hline White filling & 0.48 & Moderate & White filling & -0.00 & None \\
\hline Silver crown & 0.34 & Fair & Silver crown & 0.11 & Slight \\
\hline RCT and crown & 0.52 & Moderate & RCT and crown & 0.21 & Fair \\
\hline Gap & 0.32 & Fair & Gap & 0.12 & Slight \\
\hline Gap closes & 0.32 & Fair & Gap closes & -0.08 & None \\
\hline Bridge & 0.18 & Slight & Bridge & -0.023 & None \\
\hline Implant & 0.27 & Fair & Implant & 0.10 & Slight \\
\hline Denture & 0.18 & Slight & Denture & 0.02 & Slight \\
\hline
\end{tabular}

Mean utility values for the different health states generated through the VAS and TTO data are reported in Table 3 and 4 . A white filling generated the highest utility ( 0.786 [VAS], 0.763 [TTO]). The open gap state generated the lowest utility ( 0.344 [VAS], 0.523 [TTO]).

\section{Statistical analysis of demographic responses}

Exploratory analysis of the impact of patient characteristics on preferences is reported in Additional File 2.

\section{Discussion}

This study provides preference data on a set of tooth states capturing the most commonly occurring outcomes of intervention on cFPMs over the lifetime of the patient. The scope of health states valued is wider than previous studies and provides a consistently measured set of utility values to inform economic evaluation of treatments for cFPMs. Crucially, preference data for both a closed and open gap was provided, allowing comparison of treatment pathways which include restoration of the tooth with those that include dental extraction. These data provide utility values to support cost-utility analysis of many different treatments for molar teeth, provided that certain assumptions are met.

The data presented could be used to weight time in each of the eight health states and summarize the overall benefit to the patient in quality adjusted tooth-years (QATYs). The use of QATYs as a measure of utility requires a number of strong assumptions. The assumptions underpinning the use of QALYs apply to the use of a QATY: utility is a linear function of duration (of life or the tooth); utility is the product of duration and quality of life (or tooth) in that health state; and that lifetime utility is the sum of that associated with different health states (16). However, there are further assumptions required. Utility associated with the relevant tooth must be independent of utility associated with any other aspect of the relevant person's health during the period of evaluation. An assumption that the utility associated with the relevant tooth is independent of that arising from other health domains, such as anxiety and depression, is not dissimilar to the assumptions underpinning additive models of utility (4). However, the use of QATYs as a measure of utility requires an assumption that utility derived from the index tooth is independent of any other teeth in the person's mouth. Clearly, this is only tenable if the person maintains good overall oral health.

Preference ordering from mean VAS scores showed better correlation with health state rankings than preference ordering from mean TTO scores. This would suggest the VAS scores show better reliability than the TTO scores. The application of the TTO exercise to the valuation of a single tooth state was more challenging than the application of the VAS exercise. An open gap was selected as an anchor state and a time period of ten years was chosen after which patients would be left with an open gap (analogous to death after ten years in a TTO valuing overall health). Approximately half of respondents ranked an open gap as the worst outcome, slightly more than those that chose a denture. It is possible to elicit values for health states considered worse than the anchor state in a TTO, but we did not attempt this. Some participants required extra guidance to complete the TTO, in contrast to the ranking exercise and VAS exercises. Preferences from TTO are generally regarded as more valid than those elicited with VAS and TTO has been the 
method of choice for valuing EQ-5D-5L responses. However, this presumes the respondent has fully understood the TTO exercise. In our view it is more challenging to create plausible valuation scenarios for elicitation of TTO values for tooth health states and this would militate for the use of VAS to measure preferences despite its well-known limitations.

A few previous studies have sought to measure patient preferences over tooth states (Table 5). One of the earliest attempts used SG to elicit values from dental professionals and the general public for a posterior tooth in different states of decay and restoration (10). General public values for a filled tooth were similar to values observed in the present study; dental professionals' values were higher. Balevi \& Sheppard applied SG to elicit utilities for a root treated tooth, a fixed bridge, a removable partial denture and an implant supported crown (17). Values for a bridge and implant were a little higher than observed in the current study (18). Utilities generated using SG from the general public have been reported for extraction and root canal treatment (11).

Other researchers have sought to value health states representing multiple teeth. Values from the general public in the UK and Iran were elicited using VAS for 19 health states in which one or more teeth were missing (19). Values for a missing tooth decreased as the missing tooth varied from a posterior tooth to a central incisor. Values for a shortened dental arch decreased as the number of missing teeth increased. Values were low, even for a shortened dental arch missing second molar teeth ( 0.37 UK sample), suggesting the general public does not view a shortened dental arch as an acceptable outcome. Further work by this team using VAS indicated that prosthetic replacement is preferable to a shortened dental arch, with implant-supported prostheses were viewed as superior to removable dentures but inferior to fixed bridges (20). Similar findings have been observed in Japan in a comparison of treatment options for missing lower first and second molars (21). Current data suggests implant supported single crowns are viewed as similar or superior to a fixed bridge. There is some evidence to indicate dentists and the general public hold similar preferences when elicited by VAS (22), albeit professionals place a higher value on implant-supported prostheses than the general public (21).

The main limitation of the current study is the small sample size and the use of a convenience sample. Respondents were recruited from patients attending a paediatric dental clinic at St Thomas' Hospital, London. These respondents were chosen as a sample of the general public. Half of the eligible respondents completed the survey and demographics would indicate they are broadly representative of the local population. Data was also lacking on measures of deprivation and it was not possible to assess this aspect of representativeness. Application of these preferences to UK decision-making requires an assumption that results are generalizable.

\section{Conclusions}

In conclusion, preferences for eight dental health states spanning the management pathway for a cFPM over the lifetime of the patient have been elicited using TTO and VAS. With certain assumptions, these data allow estimation of the utility associated with the treatment alternatives, extraction or restoration, for $\mathrm{CFPM}(\mathrm{s})$ in a juvenile mouth and hence support an economic evaluation of treatment options reflecting patient preferences. Preferences elicited using VAS appear to have greater validity than those elicited with TTO and indicate a white filling and an open gap are ranked highest and lowest, respectively. This data adds to a nascent literature on health state utility values for dental health states and will support future cost-utility analyses of dental treatments.

\section{Abbreviations}

cFPM: compromised first permanent molar

VAS: visual analogue scale

TTO: time trade off

QALY: quality-adjusted life-year

QoL: quality of life

SG: standard gamble

QATY: quality adjusted tooth-year 
RCT: root canal treatment

HCRW: Health and Care Research Wales

\section{Declarations}

\section{Ethics approval and consent to participate:}

Ethical approval was gained by NHS research ethics, namely HRA and Health and Care Research Wales (HCRW) (REC reference 19/NS/0164). Written consent for participation was also gained.

\section{Consent for publication:}

Not applicable

\section{Availability of data and materials:}

The datasets used and/or analysed during the current study are available from the corresponding author on reasonable request.

\section{Competing interests:}

The authors declare no conflict of interest.

\section{Funding:}

No application for external funding was made. The study was sponsored by King's College London. The co-sponsor was NHS Guy's and St Thomas' NHS Foundation Trust.

\section{Authors' contributions:}

AC developed the questionnaire, collected and analysed the data and contributed in the writing of the manuscript. RS collected the data and contributed to the writing of the manuscript. AN developed the questionnaire and contributed to the writing of the manuscript. MTH and AB contributed to the writing of the manuscript. FB supported the statistical analysis of the data. MP developed the questionnaire and contributed in the writing of the manuscript.

\section{Acknowledgements:}

This study is part of the Children Experiencing Dental Anxiety: Collaboration on Research and Education (CEDACORE) research group.

\section{References}

1. Coast J, Smith RD, Lorgelly P. Welfarism, extra-welfarism and capability: The spread of ideas in health economics. Soc Sci Med [Internet]. 2008 Oct [cited 2020 Oct 15];67(7):1190-8.

2. Vernazza CR. The monetary value of oral health: willingness to pay for treatment and prevention [Internet]. Newcastle University; 2010 [cited 2020 Oct 15]. Available from: http://theses.ncl.ac.uk/jspui/handle/10443/1202

3. Phillips C. What is a QALY? Health economics [Internet]. 2009 [cited 2020 Oct 15]. Available from:

4. Feeny D. The Multi-attribute Utility Approach to Assessing Health-related Quality of Life. 2012 [cited 2020 Oct 15]; Available from: https://ideas.repec.org/h/elg/eechap/14021_36.html

5. EQ-5D-5L - EQ-5D [Internet]. 2017 [cited 2020 Nov 10]. Available from: https://euroqol.org/eq-5d-instruments/eq-5d-5l-about/

6. Brennan DS, Spencer A. Dimensions of oral health related quality of life measured by EQ-5D + and OHIP-14. Health Qual Life Outcomes [Internet]. 2004 Jul 13 [cited 2018 Dec 3];2(1):35.

7. Oppe M, Devlin NJ, Van Hout B, Krabbe PFM, De Charro F. A program of methodological research to arrive at the new international eq-5d-5I valuation protocol. Value Heal [Internet]. 2014 [cited 2020 Oct 26];17(4):445-53. 
8. Birch S. Measuring dental health: improvements on the DMF index - PubMed. Community Dent Heal [Internet]. 1986 Dec [cited 2020 Oct 15];3(4):303-11.

9. Antczak-Bouckoms AA, Weinstein MC. Cost-effectiveness Analysis of Periodontal Disease Control. J Dent Res. 1987;66(11):1630-5.

10. Fyffe HE, Kay EJ. Assessment of dental health state utilities. Community Dent Oral Epidemiol [Internet]. 1992 Oct [cited 2019 Jul 21];20(5):269-73.

11. Ismail Al, Birch S, Sohn W, Lepkowski JM, Belli RF. Utilities of dentin regeneration among insured and uninsured adults. Community Dent Oral Epidemiol. 2004 Feb;32(1):55-66.

12. Taylor GD, Pearce KF, Vernazza CR. Management of compromised first permanent molars in children: Cross-Sectional analysis of attitudes of UK general dental practitioners and specialists in paediatric dentistry. Int J Paediatr Dent [Internet]. 2019 Feb 14 [cited 2019 Mar 17]; Available from: http://doi.wiley.com/10.1111/ipd.12469

13. Cobourne MT, Williams A, Harrison M. National clinical guidelines for the extraction of first permanent molars in children. Br Dent J [Internet]. 2014 Dec 5 [cited 2019 Mar 16];217(11):643-8.

14. Jälevik B, Möller M. Evaluation of spontaneous space closure and development of permanent dentition after extraction of hypomineralized permanent first molars. Int J Paediatr Dent [Internet]. 2007 Sep [cited 2019 Mar 17];17(5):328-35.

15. Brazier J, Ratcliffe J, Saloman J, Tsuchiya A. Measuring and valuing health benefits for economic evaluation [Internet]. 2016 [cited 2019 Jan 14]. 348 p. Available from: https://books.google.co.uk/books? $\mathrm{hl}=$ en\&lr=\&id=r3R4DQAAQBAJ\&oi=fnd\&pg=PP1\&dq=measuring+and+valuing+health+benefits+for+economic+evaluation\&ots=yaXyRUHZp\&sig=VcNqXTI3HU0qdtoH5s1-qr8pya8\#v=onepage\&q=measuring and valuing health benefits for economic evaluat

16. Miyamoto JM. Quality-Adjusted Life Years (QALY) Utility Models under Expected Utility and Rank Dependent Utility Assumptions. J Math Psychol [Internet]. 1999 [cited 2020 Oct 26];43(2):201-37.

17. Balevi B, Shepperd S. The management of an endodontically abscessed tooth: Patient health state utility, decision-tree and economic analysis. BMC Oral Health. 2007;7:17.

18. Lewis JM. Improving dental health status indicators for evaluation. Community Dent Oral Epidemiol [Internet]. 1996 Feb [cited 2019 Nov 24];24(1):32-6.

19. Nassani MZ, Locker D, Elmesallati AA, Devlin H, Mohammadi TM, Hajizamani A, et al. Dental health state utility values associated with tooth loss in two contrasting cultures. J Oral Rehabil [Internet]. 2009 Aug [cited 2020 Oct 15];36(8):601-9.

20. Nassani MZ, Devlin H, McCord JF, Kay EJ. The shortened dental arch - An assessment of patients' dental health state utility values. Int Dent J [Internet]. 2005 [cited 2020 Oct 15];55(5):307-12.

21. Ikebe K, Hazeyama T, Kagawa R, Matsuda K, Maeda Y. Subjective values of different treatments for missing molars in older Japanese. J Oral Rehabil [Internet]. 2010 Dec [cited 2020 Oct 15];37(12):892-9.

22. Kay EJ, Nassani MZ, Aswad M, Abdelkader RS, Tarakji B. The disutility of tooth loss: A comparison of patient and professional values. J Public Health Dent [Internet]. 2014 [cited 2020 Oct 15];74(2):89-92.

23. Chestnutt IG, Hutchings S, Playle R, Morgan-Trimmer S, Fitzsimmons D, Aawar N, et al. Seal or Varnish? A randomised controlled trial to determine the relative cost and effectiveness of pit and fissure sealant and fluoride varnish in preventing dental decay. Health Technol Assess (Rockv) [Internet]. 2017 Apr [cited 2019 Jan 14];21(21):1-256.

24. Nguyen TM, Tonmukayakul U, Warren E, Cartwright S, Liew D. A Markov cost-effective analysis of biannual fluoride varnish for preventing dental caries in permanent teeth over a 70-year time horizon. Heal Promot J Aust [Internet]. 2019 Aug 27 [cited 2019 Nov 24];hpja.283.

\section{Tables}

Table 5 is not available with this version

\section{Figures}




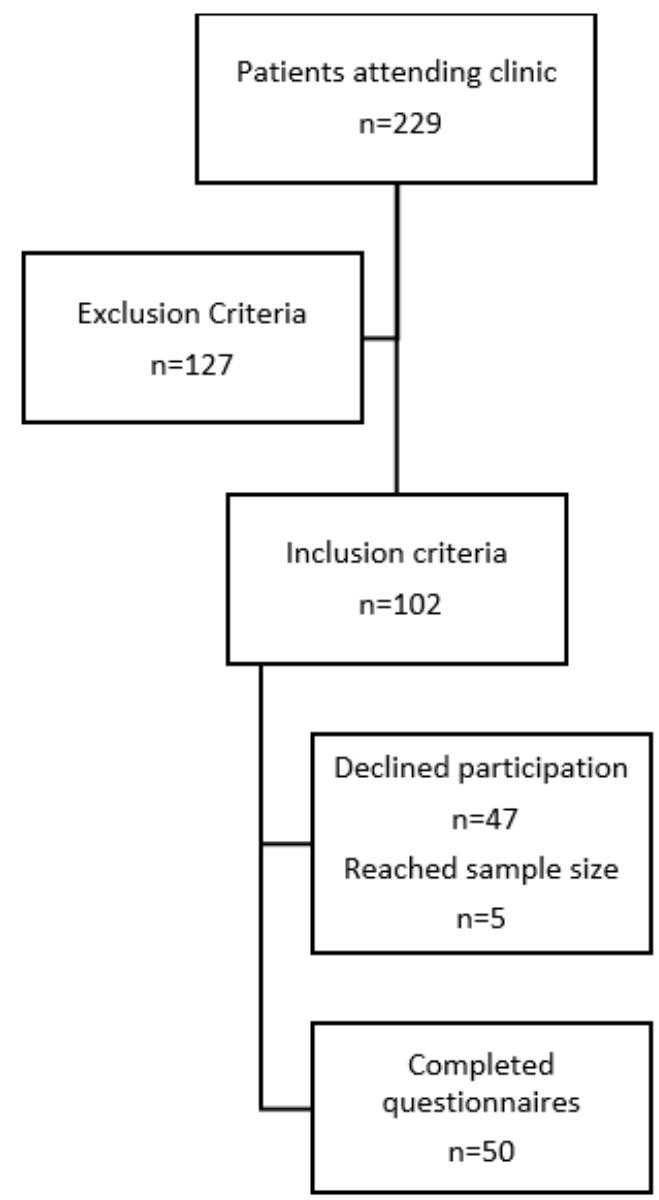

Figure 1

Flowchart of patient recruitment 


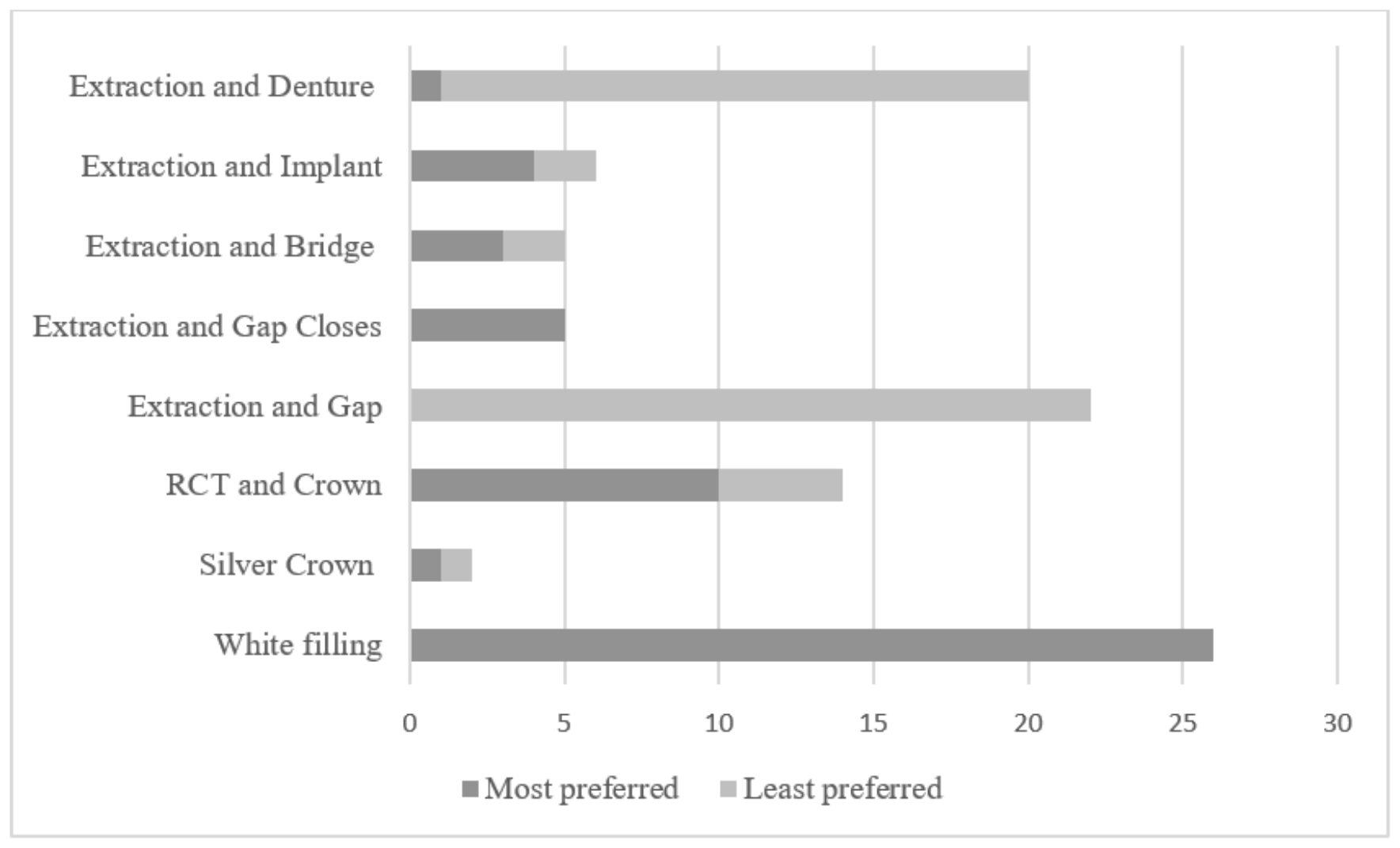

Figure 2

Ranking of most preferred and least preferred option

\begin{tabular}{|c|c|c|c|c|c|c|c|c|}
\hline & $\begin{array}{c}\text { White } \\
\text { Filling }\end{array}$ & $\begin{array}{c}\text { Silver } \\
\text { Crown }\end{array}$ & $\begin{array}{c}\text { RCT and } \\
\text { Crown }\end{array}$ & $\begin{array}{c}\text { Extraction } \\
\text { and Gap }\end{array}$ & $\begin{array}{c}\text { Extraction and } \\
\text { Gap Closes }\end{array}$ & $\begin{array}{c}\text { Extraction } \\
\text { and Bridge }\end{array}$ & $\begin{array}{c}\text { Extraction } \\
\text { and Implant }\end{array}$ & $\begin{array}{c}\text { Extraction } \\
\text { and Denture }\end{array}$ \\
\hline $\begin{array}{c}\text { Mean } \\
\text { Rank }\end{array}$ & 2.2 & 3.7 & 3.5 & 6.4 & 4.4 & 4.4 & 4.7 & 6.7 \\
\hline
\end{tabular}

Figure 3

Mean rank of each health state

\section{Supplementary Files}

This is a list of supplementary files associated with this preprint. Click to download.

- AdditionalFile1 to3.docx 